Meta

Journal des traducteurs

Translators' Journal

\title{
Vocabulaire juridique et schémas d'arguments juridiques
}

\section{Pierre Lerat}

Volume 47, numéro 2, juin 2002

Traduction et terminologie juridiques

URI : https://id.erudit.org/iderudit/008005ar

DOI : https://doi.org/10.7202/008005ar

Aller au sommaire du numéro

Éditeur(s)

Les Presses de l'Université de Montréal

ISSN

0026-0452 (imprimé)

1492-1421 (numérique)

Découvrir la revue

Citer cet article

Lerat, P. (2002). Vocabulaire juridique et schémas d'arguments juridiques. Meta, 47(2), 155-162. https://doi.org/10.7202/008005ar

\section{Résumé de l'article}

Les principales difficultés de la traduction juridique sont les dénominations, les constructions et les compatibilités sémantiques. Une méthodologie issue de Zellig Harris offre un cadre approprié : les "schémas d'arguments " (expressions prédicatives et classes d'objets), avec des applications aux constructions (le " figement ») et à la dérivation (la " condensation de schémas »). Le dictionnaire juridique français (Laboratoire de linguistique informatique, Paris 13, dir. G. Gross), avec des équivalents en anglais, utilise cette approche.
Ce document est protégé par la loi sur le droit d'auteur. L'utilisation des services d'Érudit (y compris la reproduction) est assujettie à sa politique d'utilisation que vous pouvez consulter en ligne.

https://apropos.erudit.org/fr/usagers/politique-dutilisation/ 


\title{
Vocabulaire juridique et schémas d'arguments juridiques
}

\author{
PIERRE LERAT \\ Université Paris XIII, Paris, France
}

\begin{abstract}
RÉSUMÉ
Les principales difficultés de la traduction juridique sont les dénominations, les constructions et les compatibilités sémantiques. Une méthodologie issue de Zellig Harris offre un cadre approprié : les «schémas d'arguments 》 (expressions prédicatives et classes d'objets), avec des applications aux constructions (le «figement») et à la dérivation (la «condensation de schémas»). Le dictionnaire juridique français (Laboratoire de linguistique informatique, Paris 13, dir. G. Gross), avec des équivalents en anglais, utilise cette approche.
\end{abstract}

\section{ABSTRACT}

The main difficulties in legal translation are names, constructions and semantic compatibilities. A methodology inspired by Zellig Harris offers an appropriate framework: "schemata of arguments" (predicative expressions and classes of objects), with applications to constructions ("frozen phrases") and to derivation ("condensation of schemata"). The French Legal Dictionary (Laboratoire de linguistique informatique, Paris 13, dir. G. Gross), with equivalents in English, uses this approach.

\section{MOTS-CLÉS/KEYWORDS}

schéma d'arguments, classe d'objets, dérivation, condensation, lexicographie juridique

\section{Les difficultés de la traduction juridique}

L'importance des conditions culturelles préalables en la matière est assez connue pour que je n'y insiste pas. Elle fournit des arguments dignes de considération à la thèse (défendue par d'éminents traductologues) selon laquelle avec une bonne connaissance de deux langues et une forte culture générale le reste sera donné par surcroît.

L'intérêt de travailler à un outil d'aide à la traduction et à la rédaction spécialisées pour des agents économiques ordinaires (d'entreprises et d'administrations) et pour des personnes en formation est de devoir rendre explicites des faits de langue spécialisée. On s'aperçoit alors de l'absence d'évidence pour le «non-initié».

$1^{\text {re }}$ difficulté: les dénominations

Si l'on partage l'avis de Sager pour qui « un terme est une variété fonctionnelle du nom commun» (2000: 53), la connaissance des termes est affaire d'expérience des choses et des concepts dénommés par des noms. Mais si l'on admet qu'abrogation est un terme puisque c'est un mot juridique «fonctionnel», on voit mal pourquoi abroger n'en serait pas un également (ainsi, d'ailleurs, qu'abrogatif et abrogatoire). Disons qu'abroger est un mot spécialisé, même si (voir Lerat 2001) les spécialistes du domaine auteurs de dictionnaires $(V, Q 1$, par exemple) croient avoir assez fait en traitant du nom d'action sans prendre en compte le verbe. Linguistiquement, ce sont deux 
expressions prédicatives équivalentes (avec les mêmes arguments), deux dénominations différentes d'un même concept. En outre, et surtout, les textes juridiques sont remplis de noms propres, d'expressions mixtes (comme Traité de Rome), d'abréviations, de sigles plus ou moins ésotériques. Bref, l'encyclopédie mord sérieusement sur la langue spécialisée.

\section{$2^{\mathrm{e}}$ difficulté: l'inventaire des formes}

La question de la féminisation lexicale fait sauter aux yeux le déficit de féminins juridiques dans les textes. Ce qui est moins voyant mais tout aussi réel est la défectivité morphologique dans les conjugaisons. Ainsi, la forme usuelle du verbe juridique échoir est échu, qui fait à juste titre l'objet d'une entrée dans $P$ et dans $L S$; en cherchant bien, on peut encore attester échet (Code pénal) et échoient (S982), mais ce sont des survivances dont la rareté ne doit pas faire négliger échu (or les adjectifs, participiaux surtout, sont aussi des oubliés des dictionnaires de spécialistes).

\section{$3^{\text {e }}$ difficulté: les constructions}

Il ne va pas de soi, pour un non francophone, que le sens de réputé est particulier dans la construction "être réputé + attribut», ou que l'on dit plutôt «être tenu de + infinitif» et «être tenu à + groupe nominal» etc.

$4^{\text {e }}$ difficulté : les compatibilités sémantiques

Les inventaires de cooccurrences, collocations ou phraséologismes sont des tentatives empiriques pour rendre compte de tendances. Il est souvent possible d'aller jusqu'à des règles (locales) et des exceptions (explicites); ainsi, selon $V$, abrogation se dit seulement d'un texte législatif, mais il peut aussi s'agir de la suppression d'un règlement selon Q1, et dans $S 359 a$ il est question de l'abrogation d'un contrat-type de travail, ce qui invite à considérer que l'enjeu est plus largement un texte normatif. C'est le souci de formaliser les limites de telles régularités qui plaide tout particulièrement en faveur du modèle d'analyse qui va être présenté ci-dessous.

$5^{\text {e }}$ difficulté: les reformulations

«Le consentement se présume» (S176), «le consentement est présumé» (le passif est utilisé dans le texte allemand: wird vermutet), «on présume le consentement» (moins «naturel» ?). La sécurité linguistique du rédacteur et du traducteur, en pareil cas, passe nécessairement par des références textuelles. Ce scrupule philologique était l'une des particularités de $L S$. Il est encore accru dans le fichier qui va être présenté plus loin, comme le montre la prédominance des abréviations de textes validés par des autorités politiques nationales et européennes.

\section{Apports de la linguistique générale}

La conception du fichier réalisé dans le cadre du Laboratoire de linguistique informatique de Paris 13 - Villetaneuse doit beaucoup à une approche générale des types d'emplois des unités lexicales. Cette méthodologie a fait l'objet de diverses présentations, notamment dans le $\mathrm{n}^{\circ} 131$ de la revue Langages (1998) et dans le $\mathrm{n}^{\circ} 46-1$ de Meta (2001), et sa partie descriptive la plus connue est l'élaboration de «classes d'objets» 
sémantico-syntaxiques. Je la résume à grands traits et je propose une contribution inspirée de cette théorie, pour rendre compte des dérivés spécialisés.

\subsection{Les schémas d'arguments}

Entre le niveau très particulier du mot et le niveau très général de la phrase, un niveau d'analyse particulièrement fécond est celui de la phrase simple réduite à un schéma propositionnel dont le cœur est une expression prédicative autour de laquelle se distribuent des arguments (sujet et objets). On peut reconnaître ici le schéma de Z. Harris ("opérateur et arguments») issu d'une tradition de logique mathématique qui remonte à Frege (voir Le Pesant et Mathieu-Colas 1998). Ce petit brin d'épistémologie n'est là que pour situer une filiation (qui en France passe par Maurice Gross, informaticien et linguiste) et inciter les juristes et traducteurs qui ont fait l'effort de s'habituer à un (autre) schéma de phrase élémentaire, "SN + SV", à la suite de la «linguistique cartésienne» (Chomsky compris), à le remplacer dans leur esprit par un schéma « $\mathrm{V}\left(\mathrm{N}^{\circ}, \mathrm{N} 1\right)$ ». Ce changement d'approche sera justifié si les résultats sont convaincants - et eux seuls comptent en l'occurrence.

\subsection{Les expressions prédicatives}

Contrairement au logicien, le linguiste s'intéresse moins aux prédicats (concepts en tant qu'unités de pensée) qu'aux expressions prédicatives (formulations dans telle langue). Une expression prédicative (verbale, nominale ou adjectivale) se reconnaît à ce qu'elle a besoin d'objets pour être syntaxiquement complète, de même qu'un prédicat logique a besoin d'être «saturé» par des arguments.

Ex.: Ils louent un pavillon, mais aussi Ils sont en location dans un pavillon et également Ils sont locataires d'un pavillon.

Les verbes prédicatifs ont une ou plusieurs constructions canoniques, qui se réalise(nt) totalement ou partiellement dans le discours.

Ex. 1 : Ils louent un pavillon à un commerçant pour un loyer de tant / Ils louent à un commerçant (construction acceptant l'économie du complément d'objet direct).

Ex. 2: Ils ont enfin contracté (construction intransitive complète) / Ils ont contracté un engagement / une dette / une obligation (construction transitive directe n'autorisant pas d'ellipse).

Les noms prédicatifs ont besoin de verbes particuliers pour être construits. Ce sont des verbes «supports».

Ex: prendre effet, donner congé, faire grève.

Les adjectifs prédicatifs (en gros, les «qualificatifs» par opposition aux «adjectifs de relation ») ont besoin d'un verbe copule (être) pour être au cœur d'une phrase simple. Ils ont un argument (comme non avenu) ou deux (comme responsable de).

\subsection{Les classes d'objets}

Les systèmes de traduction automatique utilisent des filtres sémantico-syntaxiques tels que les traits généraux ACTION, ÉTAT, ÉVÉNEMENT, HUMAIN, CONCRET 
etc. qui suffisent quelquefois à caractériser un mot ou un usage d'un mot polysémique. Le vocabulaire juridique se prête comme les autres à de tels repères: résiliation est un nom d'action, usufruit un nom d'état, catastrophe naturelle un nom d'événement.

Il est clair toutefois qu'ABSTRAIT, par exemple, est valable à propos de presque tous les mots juridiques. Il faut donc disposer de classes moins générales, parentes sémantiquement, comme <somme d'argent $>$ (par convention, au LLI, les chevrons entourent les noms de classes d'objets). Pour les établir, on se sert de la combinatoire lexicale (pour un substantif, les verbes appropriés).

Ex. : une <somme d'argent> est quelque chose que l'on peut à la fois devoir / payer / percevoir / rembourser / virer.

La combinaison des traits généraux et des classes d'objets est quelquefois nécessaire.

Ex. $1: \mathrm{N}<$ HUMAIN, automate $>$ remplir $<$ formulaire $>$ (Le Pesant et Mathieu-Colas 1998: 29).

Ex. 2: N < HUMAIN> actionner / arrêter / réparer / régler <machine> (cf. Buvet 1997: 13), ce qui est remarquable car en dehors de cet emploi technologique chacun des verbes pris séparément entre par ailleurs dans un emploi juridique au moins: on peut actionner un $<$ HUMAIN $>$ (individuel ou collectif), arrêter un $<$ HUMAIN $>$ (individuel) ou, dans un autre sens, une $<$ décision $>$, réparer un $<$ préjudice $>$ ou une $<$ faute juridique $>$ et régler un $<$ différend $>$.

\subsection{Les degrés de figement}

Cette notion développée par G. Gross (1996) fournit une échelle syntaxique, distributionnelle (insertions possibles) et transformationnelle (reformulations possibles).

Ex. 1: on peut fixer, entre autres compléments possibles, un délai, mais ce qui est imparti ne peut être qu'un délai.

Ex. 2: en vertu de est plus figé que à titre de car on peut dire à ce titre mais non ${ }^{*}$ en cette vertu.

\subsection{Les condensations de schémas d'arguments}

Cette idée résulte d'un retour critique sur celle de "paradigme dérivationnel» empruntée à Guilbert et présentée dans mon livre de 1995 comme particulièrement propre à rendre compte de "séries terminologiques» de dérivés telles que louer // loueur // locataire // loyer. Il s'agissait pour moi comme pour Guilbert de la « série des réalisations syntaxiques différentes d'un même noyau sémantique» (p. 176), ce qui intuitivement n'était pas faux mais laissait à désirer techniquement:

- partir d'un schéma de phrase « $\mathrm{SN}+\mathrm{SV}$ » est de peu d'intérêt pour une expression prédicative telle que louer, qui se caractérise par un jeu complexe d'arguments: $\mathrm{N}<$ HUMAIN $>$ louer $\mathrm{N}<$ habitation $>$ à $\mathrm{N}<$ HUMAIN $>$ contre <somme d'argent $>$ (cf. G. Gross 1998: 108);

- l'approche générativiste de Guilbert ne permet pas d'exclure des mots virtuels dont le traducteur n'a que faire $\left({ }^{*}\right.$ locateur, par exemple);

- l'intuition d'un «noyau sémantique» n'a pas de contrepartie combinatoire vérifiable, contrairement aux classes d'objets. 
Si l'on considère que tout emploi d'un mot spécialisé renvoie à un scénario de réalité lexicalisé au moyen d'un schéma d'arguments, ce qui est maintenant ma thèse, l'implicitation d'arguments ou même du prédicat apparaît comme un procédé économique normal dans la communication, notamment dans l'anaphore: le loyer de l'appartement, le locataire de la villa, ou même le loyer, le locataire (anaphores exclues dans le cas d'une location de voiture, qui correspond à un autre scénario que la location d'un $<$ bien immeuble $>$ ).

Un même dérivé peut condenser des schémas d'arguments différents, et donc se définir diversement.

Ex. : $\mathrm{N}<$ HUMAIN $>$ être en $<$ ETAT: retraite $>\rightarrow$ retraité

$\mathrm{N}<$ HUMAIN $>$ percevoir $<$ somme d'argent $:$ retraite $>\rightarrow$ retraité

Cette approche des dérivés spécialisés en termes de condensés de schémas d'arguments rend compte en particulier de l'abondance des nominalisations dans la communication entre initiés: les arguments requis peuvent alors rester implicites.

\section{Principes de lexicographie juridique bilingue}

La façon de faire décrite ci-dessous tient compte des acquis de l'expérience d'autrui et n'est originale que par l'application de la méthodologie du Laboratoire de linguistique informatique au vocabulaire juridique (au sens large). Au demeurant, l'idée selon laquelle un dictionnaire bilingue spécialisé ne peut être qu'unidirectionnel est supposée acquise (voir LS: 4). En outre, pour ce qui est de la macrostructure, il ressort de ce qui précède que font l'objet d'entrées les dénominations de concepts auxquels sont associables des effets de droit (dans la vie économique, sociale, politique ou administrative), que ces dénominations sont lemmatisées si le singulier ou l'infinitif sont usuels et que les expressions totalement figées font également partie de la nomenclature. C'est donc sur la microstructure que je vais m'attarder davantage, car c'est elle qui est innovante.

\subsection{Des schémas d'arguments spécialisés canoniques}

Un schéma d'arguments prend dans un texte des formes variées. Ainsi, il est rare que l'on trouve impartir un délai. Par exemple, dans le Traité d'Amsterdam (A149), ce qui est écrit est en français délais impartis et en anglais time limits laid. Ce qui est pris en compte dans le dictionnaire n'est pas une telle séquence observable mais en quelque sorte sa matrice, à savoir impartir un délai et to lay a time limit, respectivement.

Il s'agit d'un schéma syntaxique, donc pour un nom prédicatif on indiquera son verbe support.

Ex : <fonction juridique: mandant $>$ donner mandat à $<$ fonction juridique: mandataire $>$.

Dans cet exemple l'absence d'article entre le verbe et le nom est notée; le plus souvent, c'est un article indéfini qui exprime la plus grande généralité, de même que l'infinitif présent.

\subsection{Des références textuelles spécialisées}

Le principe de traçabilité est aussi ancien que la philologie. Un emploi est validé par des autorités, de préférence plurielles, la visée étant ici descriptive plutôt que normative. 
Ainsi, il n'y a pas à arbitrer entre les autorités européennes référencées ci-dessous pour traduire en anglais rendre un jugement: l'important est que l'offre soit multiple et validée. Le choix offert sera, jusqu'à plus ample informé, entre to give judgement (A35), to pronounce a judgement (DH6) et to render a judgement (DH27). De même, pour traduire en allemand subrogation, il est utile au traducteur de savoir que dans le Code civil de l'Allemagne on dit Abtretung, qu'en Suisse on utilise Übergang (S149) et qu'à la Cour de Justice de Luxembourg une expression concurrente est Rechtsnachfolge (voir Lerat et Sourioux 1994: 173).

\subsection{Les figements}

Les degrés de figement déterminent des enchaînements plus ou moins contraints. Ainsi, sans préjudice de est entièrement figé et mérite une entrée propre (son sens n'étant pas compositionnel), tandis que en qualité de a sa place sous qualité (son sens est compositionnel et il est possible de dire en sa qualité de). Les dénominations longues méritent de figurer comme sous-entrées si elles sont statistiquement courantes: un dégât des eaux est un dégât parmi d'autres, mais bénéficiant d'une spécificité en matière d'assurances (il en va de même pour catastrophe naturelle). Pour les locutions verbales, c'est aussi la fréquence qui fait choisir prendre des dispositions plutôt que prendre une disposition, mais le figement porte sur l'attente du verbe support, qui n'est pas prédictible (ainsi, en allemand, l'équivalent est Bestimmungen treffen).

\subsection{Les condensés}

Le caractère «abstrait» d'un nom d'action doctrinal tel que disposant résulte de l'économie de moyens par laquelle l'initié est renvoyé implicitement à un schéma où un $<$ HUMAIN $>$ dispose de $<$ bien $>$ en faveur de $<$ HUMAIN $>$. En ce sens l'équivalent en. settlor (LS) est plus abstrait que en. party disposing (Q2) ou que de. Verfügender (LS), mais dans chaque cas il reste présupposé que l'enjeu est un bien.

\subsection{Les schémas d'arguments équivalents}

Le lieu de l'équivalence est la matière de débats académiques entre minimalistes (terminologues attachés aux dénominations) et maximalistes (traductologues attachés au texte global, voire à l'«intention de communication»). Le schéma d'arguments représente un niveau réaliste, contrôlable et suffisant, à tout le moins, pour lever beaucoup de polysémies.

Ex. 1: <écrit juridique $>$ disposer que $=$ en. to provide $(Q 2)=$ de. bestimmen $(S 13)=i$. prescrivere (S13).

Ex. 2: <HUMAIN $>$ disposer de $<$ bien $>=$ en. to dispose $(\mathrm{Q} 2, \mathrm{ONU})=$ de. verfügen $(S 240)=$ es. disponer $(\mathrm{ONU})=$ it. disporre $(\mathrm{S} 240)$.

\subsection{Usage documentaire des classes d'objets}

Les indications de «domaines» obéissent à une logique documentaire entièrement pragmatique (dans les limites d'un système donné). Ainsi, une recherche limitée au domaine juridique, dans EURODICAUTOM, ferait perdre beaucoup d'informations 
sur subrogation et subroger, du fait que certains emplois pertinents y sont codés « Postes» ou «Journalisme», pour des raisons qui doivent être administratives. L'usage de mentions de sous-domaines ne fait qu'accroître les aléas de la consultation (voir Lerat 2001).

Les classes d'objets peuvent être utilisées en documentation spécialisée. Ainsi, la disposition au sens du droit civil est un < acte unilatéral> parmi d'autres. Une façon d'affiner le profil juridique de la notion est d'utiliser le type d'argument requis en guise de sous-domaine fondé linguistiquement. Une façon de formaliser cette spécificité consiste à coder principalement la classe du mot traité et secondairement celle d'un argument typique.

Ex. 1 : pour disposer, <acte unilatéral $>\&<$ bien $>$ (ce qui vaut pour huit noms, de cautionnement à testament).

Ex. 2: pour travailleur, < fonction juridique $>\&<$ activité économique $>$ (ce qui vaut pour 31 noms, d'actionnaire à vacataire).

Les langues étant à vocation pluridisciplinaire (et à plus forte raison non disciplinaire), des classes d'objets gagées sur des verbes appropriés évitent les faux problèmes, comme celui de savoir si une résolution de l'ONU est «fondamentalement» juridique ou politique. Une solution satisfaisante parait être la suivante en l'occurrence: $<$ décision $>\&<$ autorité politique $>$.

\section{RÉFÉRENCES}

Buvet, P.-A. (1997): «Les noms de machines en -euse», Cahiers de lexicologie, 71, p. 5-19.

Gross, G. (1996): Les expressions figées en français, Paris, Ophrys.

Gross, G. (1998): «Pour une véritable fonction "synonymie" dans un traitement de texte», Langages, 131, p. 103-114.

Gross, G. et M. Mathieu-Colas (2001): «Description de la langue de la médecine», Meta, 461, p. 68-81.

Le Pesant, D. et M. Mathieu-Colas (1998) : «Introduction aux classes d'objets », Langages, 131, p. 6-33.

Lerat, P. (1995a): Les langues spécialisées, Paris, PUF; trad. esp. Las lenguas especializadas, Barcelona, Ariel, 1997.

Lerat, P. (1995b) : «Compétence rédactionnelle et compétence technique», Meta, 40-2, p. 244249.

LeRAT, P. (2001) : «Qu'est-ce qu'un verbe spécialisé? Le cas du droit», à paraître dans les Cahiers de lexicologie.

SAGER, J.-C. (2000) : «Pour une approche fonctionnelle de la terminologie», in H. Béjoint et Ph. Thoiron (dir.), Le sens en terminologie, Presses Universitaires de Lyon, p. 40-60.

Corpus textuels

A: Traité d'Amsterdam (version consolidée, http://europa.eu.int).

CCE: 83-84: Vocabulaire des traités européens, Commission des Communautés européennes, $3^{\mathrm{e}}$ éd., Bruxelles, 1983-1984.

DH: Convention de sauvegarde des droits de l'Homme et des libertés fondamentales, Conseil de l'Europe, 1950: http://www.coe.fr/eng/legaltext

G: Grundgesetz / Loi fondamentale (version du 22-7-98 de la constitution allemande): http:// www.bijus.org

$R C$ : Convention européenne relative à l'assurance obligatoire de la responsabilité civile en matière de véhicules automoteurs, Conseil de l'Europe, 1959: http://www.coe.fe/eng/legaltext 
S: Code des obligations (1993) / Obligationenrecht (1993) / Codice delle obbligazioni (1992), Berne, Chancellerie Fédérale.

\section{Dictionnaire}

E: EURODICAUTOM: http://eurodic.ip.lu

F: Dictionnaire de droit privé de la famille et lexiques bilingues / Private Law Dictionaries of the Family and Bilingual Lexicons (Université Mc Gill), Cowansville, Yvon Blais Inc., 1999.

LS: LERAT, Pierre et SOURIOUX, Jean-Louis (1994): Dictionnaire juridique. Terminologie du contrat. Français - anglais - allemand, Paris, Conseil international de la langue française.

ONU: Law Terminology (1990), 2 vol., Genève, ONU (eng., esp., fr).

P: Potonnier, Georges et Potonnier, Brigitte (1990): Dictionnaire de l'économie, du droit et du commerce ( $2^{\mathrm{e}}$ éd.), t. II (français - allemand), Wiesbaden, Brandstetter.

Q1: Dictionnaire de droit privé (1985): Montréal, Université McGill,

Q2: Supplément au Dictionnaire de droit privé (1988): Montréal, Université McGill.

$V$ : Vocabulaire juridique (2000): Vocabulaire juridique, dir. G. Cornu, Paris, PUF ( $8^{\mathrm{e}}$ édition).

$V D H$ : fichier (non publié) du vocabulaire des droits de l'Homme en français et en anglais

(Christine Chodkiewicz, Laboratoire de linguistique informatique, Université Paris XIII). 